\title{
ÉVOLUTION DES COMPORTEMENTS ALIMENTAIRES : ADAPTATIONS MORPHOLOGIQUES ET SENSORIELLES
}

\author{
Claude Marcel Hladik ${ }^{1}$, Patrick Pasquet ${ }^{2}$
}

Résumé. - En analysant la variabilité du comportement alimentaire des primates, nous proposons de situer les processus évolutifs, morphologiques et sensoriels, qui ont contribué à la grande diversité des régimes alimentaires de l'homme actuel. Les relations allométriques établies à partir des mesures du tractus digestif permettent une séparation fonctionnelle des formes folivores, frugivore/granivore (omnivore) et de celles dont le régime s'appuie essentiellement sur les protéines animales. Toutefois ces données ne permettent en aucun cas d'argumenter l'hypothèse selon laquelle l'encéphalisation serait corrélée à la réduction quantitative du tractus digestif. Les adaptations sensorielles, portan en particulier sur la perception gustative, constituent également une des bases de différenciation des comportements alimentaires. Elles sont discutées en tenant compte des disponibilités alimentaire dans les différents milieux et de leur évolution, en rapport avec la couverture des besoins énergétiques et l'évitement des composés toxiques. Dans cette optique, nous mettons l'accent sur l'importance de la perception de certains produits goûtant dans l'amer pour expliquer la diversité des comportements alimentaires chez l'homme.

Mots-clés : primates, allométrie, tractus digestif, bilan énergétique, encéphalisation, perception gustative.

EVOLUTIONARY ASPECTS OF FEEDING BEHAVIOR: MORPHOLOGICAL AND SENSORY ADAPTATIONS.

Summary. - Morphological and sensory evolutionary processes are presented in relation to the variability of primate feeding behavior, and discussed according to the present diversity of human diets. Digestive tract allometric relationships allow to separate classes of adaptations in relation to the major dietary components: leafy materials, fruits and seeds (omnivorous primates), and animal protein. But the hypothesis linking encephalization and the reduction of the digestive tract is not corroborated, given the data in hand. Sensory adaptations (mostly on taste sensitivity) are discussed according to energy needs and the necessity to avoid poisonous substances in various environments. In this context we focus on the variability of the perception of some bitter substances as an important determinant of feeding behavior in man.

Key words: primates, allometry, digestive tract, energy balance, encephalization, taste perception

1. E.P. 2017 du CNRS (Éco-Anthropologie: Environnements, Individus et Sociétés), Laboratoire d’Écologie Générale du MNHN. 4 avenue du Petit Château, 91800 Brunoy; e-mail : hladik@ @cr.jussieu.fr.

2. E.P. 1781 du CNRS (Dynamique de l'Évolution Humaine), 44 Rue de l'Amiral Mouchez, 75014 Paris. e-mail : pasquet@ivry.cnrs.fr 


\section{INTRODUCTION}

La diversité des comportements alimentaires des Primates représente, à l'échelle de notre temps vécu actuel, une sorte d'image figée des processus qui ont accompagné la radiation évolutive de l'Ordre auquel notre espèce se rattache. Les études comparatives portant sur ces comportements et sur les adaptations morphologiques et sensorielles dont ils dépendent, permettent de préciser certains processus évolutifs et d'expliciter la diversité des régimes alimentaires des populations humaines.

Afin d'illustrer d'exemples concrets les corrélations entre les régimes alimentaires et les adaptations morphologiques et physiologiques à différents environnements, nous rappellerons d'abord quelques résultats publiés sur les Primates non humains. Cela nous amènera vers une analyse critique de certaines théories récentes relatives à l'hominisation, en particulier celles qui concernent les équilibres entre la dépense énergétique et les apports possibles par différentes stratégies de subsistance.

Une part de la variabilité du comportement alimentaire des populations humaines peut s'expliquer en fonction des mécanismes d'adaptation à des environnements particuliers et à leur variation au cours de périodes plus ou moins récentes (parfois à l'échelle de la seule période historique), en particulier si nous prenons en compte les variations du système sensoriel. Car les récepteurs chimiosensibles des organes du goût, considérés comme partie intégrante du système alimentaire, sont impliqués, non seulement à court terme dans la régulation de la prise alimentaire mais également à plus long terme, dans les équilibres de l'alimentation des groupes humains vivant dans différents environnements.

\section{DIVERSITÉ DU COMPORTEMENT ALIMENTAIRE DES PRIMATES ACTUELS}

La comparaison du régime alimentaire d'un ensemble d'espèces de Primates vivant dans un même milieu (espèces sympatriques) permet de mettre en évidence les adaptations qui ne sont pas directement dépendantes de la composition des ressources disponibles, puisque chacune de ces espèces se trouve confrontée au même ensemble d'aliments potentiels. Par exemple, dans la forêt de Barro Colorado (Panama), cinq espèces de singes Platyrhiniens ont des comportements qui se différencient par l'utilisation de différentes quantités de fruits, de feuillages et d'insectes (Figure 1). Les observations directes de ces cinq primates, sur le terrain, pendant 18 mois (Hladik et Hladik, 1969) ont permis de quantifier à la fois les proportions des différents aliments ingérés au cours d'un cycle annuel et la variabilité dans la prise alimentaire quotidienne de chacune des espèces.

L'espèce la plus insectivore, le Tamarin à Nuque Rousse, Saguinus geoffroyi, (Figure 1A) est un animal dont le poids corporel n'excède pas 500 grammes. S'il peut exceptionnellement ingérer $100 \%$ de nourriture animale (insectes et autres invertébrés)

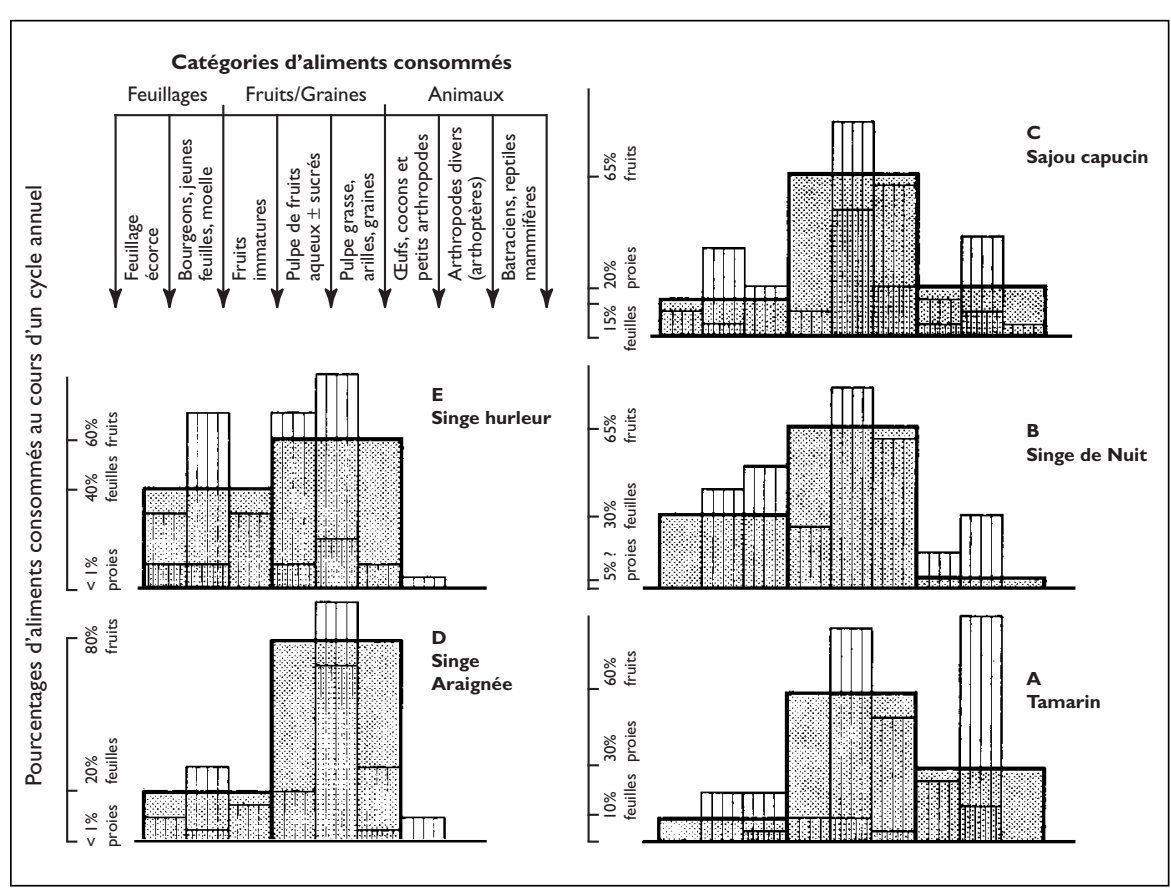

Figure 1. - Comparaison du régime alimentaire de cinq espèces de Primates de la forêt de Barro Colorado, Panama, (d'après Hladik et Hladik, 1969). Les surfaces en grisé indiquent, pour chaque espèce, les pourcentages des grandes catégories d'aliments ingérés au cours d'un cycle annuel: feuilles et autres aliments fibreux (partie grisée à gauche), fruits et graines (partie grisée au centre), insectes ou autres proies (partie grisée à droite). La variabilité de la prise alimentaire quotidienne (pourcentages maximum et minimum observés) est représentée par les colonnes superposées au reste du schéma, pour des catégories détaillées d'aliments.

pendant de courtes périodes, la moyenne annuelle de son alimentation (estimée en poids frais ingéré) inclue environ $60 \%$ de fruits. Les glucides solubles de ce régime lui apportent le complément d'énergie nécessaire à aux longs déplacements pour la recherche des invertébrés dont les lipides couvrent une large part de la dépense énergétique. Pour le Singe de Nuit, Aotus trivigatus (Figure 1B) ainsi que pour le Sajou Capucin, Cebus capucinus (Figure 1C), la proportion d'insectes et d'autres petits animaux consommés est bien moindre (respectivement $5 \%$ et $15 \%$ ), en dépit d'une stratégie alimentaire qui implique, en particulier chez le Capucin, une intense activité d'arrachage des écorces et des bois morts pour la recherche de ces proies. Mais le poids corporel relativement élevé de cette espèce ( 2 à $3 \mathrm{~kg}$ ) implique un total de prise alimentaire plus important, dans lequel la proportion de petites proies est nécessairement limitée par un rendement de 
capture qui dépend davantage du temps investi que de la taille de l'animal. On notera de même que, pour les deux autres espèces, le Singe-Araignée, Ateles geoffroyi, (Figure 1D) et le Singe Hurleur, Alouatta villosa, (Figure 1E) dont les poids corporels sont plus élevés (respectivement $5 \mathrm{~kg}$ et $10 \mathrm{~kg}$ ), un régime frugivore-folivore permet une bonne adaptation aux dépenses énergétiques, les feuillages (de l'ordre de $20 \%$ et $40 \%$, en moyennes anuelles des quantités ingérés) servent à la couverture des besoins en protéines. Dans le cas de ces espèces de grand format, le faible rendement de la chasse aux proies de petite taille ne compenserait pas la dépense énergétique.

Seule la nécessité d'apport de certains acides aminés indispensables à l'équilibre du régime peut expliquer qu'un Primate de grande taille, par exemple un Chimpanzé, consacre une grande partie de son budget-temps à la recherche et à la consommation de proies de petites taille comme les termites et les fourmis (Hladik et Viroben, 1974; Wrangham, 1977). En ce qui concerne les singes de Barro Colorado, dont les stratégies alimentaires sont présentées ci-dessus, il est apparu que les protéines des feuillages (en particulier celui des jeunes pousses de légumineuses) apportent suffisamment d'acides aminés indispensables (Hladik et al., 1971). Les pulpes des fruits permettent de couvrir l'essentiel des besoins énergétiques; cependant, pour les animaux de grand format, la proportion des feuillages sera nécessairement plus grande, en fonction d'un budget-temps qui limite la possibilité de trouver une quantité suffisante de sucres et de lipides dans les arilles et les pulpes des fruits de plantes très dispersées.

On observe ainsi, dans l'Ordre des Primates, des régimes alimentaires quasiment aussi diversifiés que pour l'ensemble des Mammifères. Cependant le régime alimentaire humain, en dépit des grandes variations observées chez différentes populations - du plus végétarien chez les Hindous au plus carné chez les Inuit —, reste dans une fourchette assez comparable aux types Sajou, Macaque ou Chimpanzé pour lesquels un mélange de nombreux produits (végétaux ou animaux) est indispensable à la couverture des besoins nutritionnels. L'exemple pré-cité des cinq espèces de Primates de Barro Colorado n'inclut pas les formes de spécialisation les plus extrêmes. En fait, on a observé, dans d'autres types de forêts (Hladik, 1981), des Primates dont le régime est totalement à base de proies animales (par exemple le genre Loris) et d'autres dont le régime comprend essentiellement des feuillages (par exemple le genre Lepilemur ainsi que les différentes espèces de la famille des Colobidae).

Parmi ces dernières formes consommatrices de feuillages, quasiment aussi spécialisées que des ruminants, deux singes du genre Presbytis vivant au Sri Lanka ont fait l'objet d'études suffisamment détaillées pour que les variations annuelles des régimes alimentaires (Figure 2) permettent de différencier les comportements et des stratégies énergétiques. Ces deux espèces, comme dans le premier exemple présenté ci-dessus, utilisent les ressources d'un même environnement ; toutefois elles ne diffèrent ni par le format (elles pèsent toutes les deux environ $10 \mathrm{~kg}$ ), ni par la morphologie du tractus digestif (Amerasinghe et al., 1971). Leurs stratégies alimentaires se différencient sur une base essentiellement comportementale: en dépit des variations saisonnières très importantes dans les quantités de fruits ou de jeunes feuilles disponibles, l'un de ces Primates, Presbytis

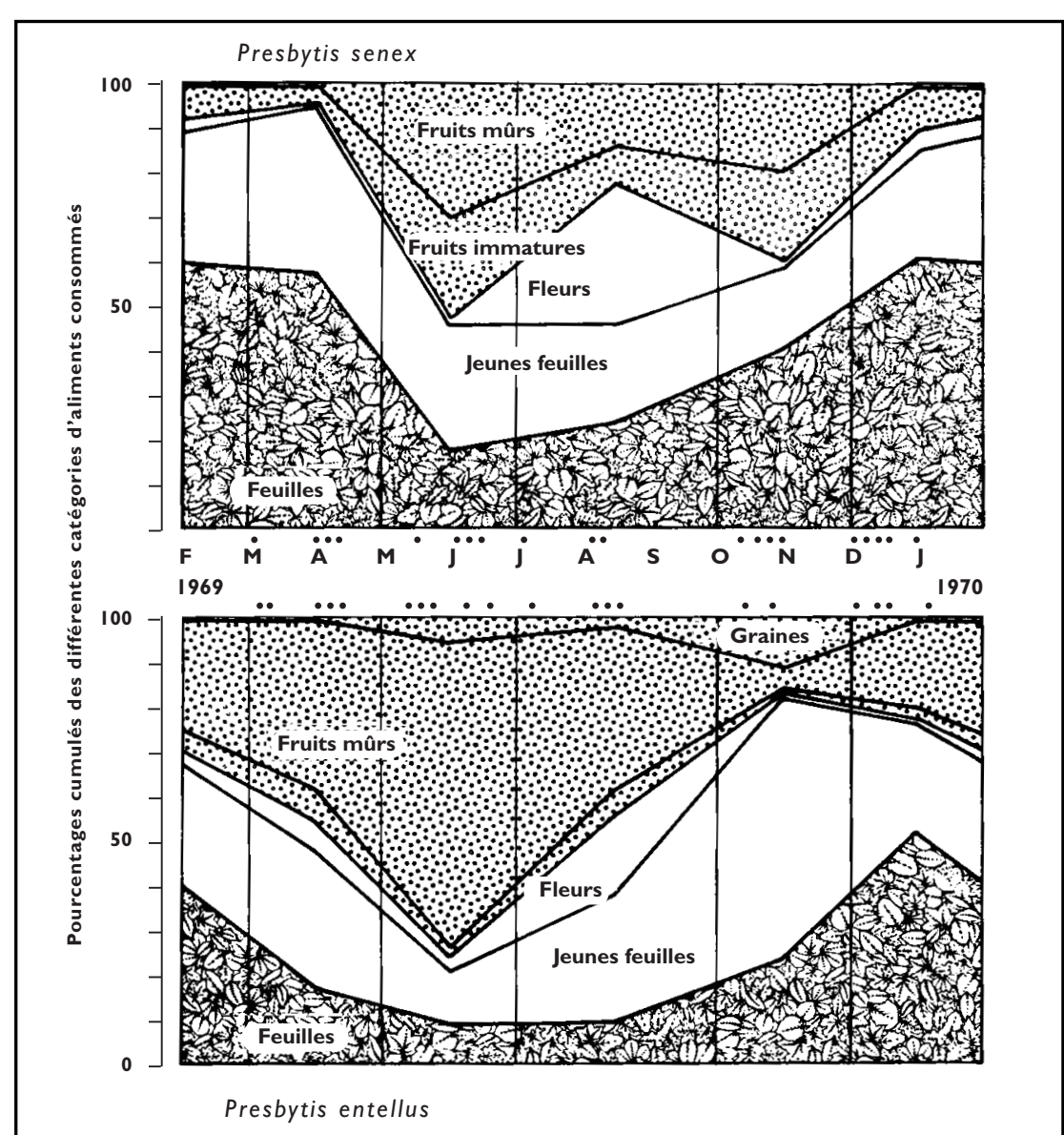

Figure 2.- Variation annuelle de la consommation de différentes catégories d'aliments par deux espèces sympatriques de Colobidae du Sri Lanka (Presbytis senex et P. entellus), en pourcentages cumulés des poids consommés au cours des différentes phases du cycle saisonnier (d'après Hladik et Hladik, 1972).

entellus, maintient, tout au long de l'année, un régime sensiblement plus riche en fruit que celui de l'autre espèce (P. senex). Ce régime qui lui apporte une plus grande quantité d'énergie nécessite d'importants déplacements afin d'utiliser un plus grand nombre de plantes disséminées sur de vastes étendues. Cette «stratégie» correspond aussi à une différence de la structure des groupes, l'espèce la moins mobile (P. senex), qui utilise les ressources les plus communes à faible densité énergétique, vivant en groupes de 4 à 7 
individus sur des territoires de moins de 4 hectares ; alors que $P$. entellus vit en groupes de 20 à 30 individus sur des territoires de 10 à 15 hectares (Hladik, 1981). Un faisceau d'arguments convergents permet d'interpréter ces deux stratégies — dont l'une implique une plus forte motivation des animaux pour la recherche des aliments les plus énergétiques - en fonction des différences de la perception gustative (voir ci-dessous) qui se maintiennent au cours des variations saisonnières (Hladik et Simmen, 1996).

La variation saisonnière du régime alimentaire peut également être très marquée dans les populations humaines vivant en économie de subsistance (Garine et Harrison 1988), dépendant essentiellement, des disponibilités saisonnières. Cependant, chez Homo sapiens, les réponses culturelles vont bien au-delà des adaptations biologiques et permettent une gestion élaborée des effets de la saisonnalité.

D'un point de vue purement biologique, deux ordres explicatifs sous-tendent les différenciations interspécifiques des comportements alimentaires: morphologique et sensoriel.

\section{ADAPTATIONS MORPHOLOGIQUES AU COMPORTEMENT ALIMENTAIRE}

\section{Primates non humains}

À l'échelle des temps où se situe l'évolution des espèces, les adaptations morphologiques à l'alimentation ont nécessairement porté sur l'ensemble de l'appareil digestif; mais il est bien évident que seules les parties dures des espèces disparues ont pu se fossiliser; et c'est sur la denture que sont réalisées la plupart des études de paléoécologie. Inversement, en ce qui concerne les parties molles du tractus digestif, seule l'anatomie comparée des espèces actuelles a permis d'avancer de nouvelles hypothèses sur les adaptations possibles des différentes radiations. Ainsi, par référence aux formes actuelles dont on connaît le régime, on peut interpréter les grandes tendances de l'évolution des espèces fossiles.

La morphologie fonctionnelle des dents a été revue par Lucas (1994) en analysant les formes du point de vue de leur effet pour deux actions complémentaire: le déchiquetage et le broyage. Cela permet de déduire l'importance des grandes catégories d'aliments utilisables avec efficacité par une espèce fossile, par comparaison de ces caractères de la denture avec ceux des espèces actuelles dont on connaît le régime. Les études portant sur l'ensemble de la mâchoire (Kay et Hylander, 1978), en particulier le rapport entre la longueur de la partie frontale (servant à la saisie et à la découpe des fruits) et celle de la partie de la denture située à l'arrière des canines (pour l'écrasement des parties dures ou fibreuses, surtout les feuillages) ont été également très utiles pour déduire les grandes catégories d'aliments auxquelles un primate était adapté.
C'est sur ces considérations des structure dentaires que de nombreux points concernant l'évolution de l'alimentation dans la lignée des Hominidés ont pu être éclaircis. Mais pour expliquer la marge de variabilité d'un régime en précisant les catégories d'aliments (par exemple pour les primates non humains présentés plus haut), l'ensemble de la morphologie du système digestif doit être prise en compte.

Pour comparer, d'un point de vue fonctionnel, l'appareil digestif de différentes espèces, il est indispensable de tenir compte des facteurs d'allométrie (rapports d'une fonction à la taille de l'animal) dont Martin (1981) a montré le poids considérable dans la radiation évolutive des primates. Rappelons que lorsque Cuvier (1805) a voulu comparer les mesures du tractus digestif de mammifères de différents formats, il n'est pas apparu de relation évidente avec les régimes alimentaires. En revanche, les mesures de la surface des muqueuses digestives rapportées au carré de la taille (tête + corps) des primates non humains (Hladik 1967), permettent de grouper les espèces dans des ensembles correspondant aux régimes folivore, frugivore et insectivore.

Les méthodes modernes de calcul des rapports d'allométrie permettent une analyse plus fine de ces mesures du tractus digestif en fonction des adaptations au régime alimentaire (Hladik, 1977; Chivers et Hladik, 1980). Certains de ces résultats (Figure 3), ont été regroupés en fonction des régimes alimentaires connus : un groupe de folivores (dont l'alimentation comprend, en moyenne, plus de $50 \%$ de feuillages), un groupe de frugivores (plus de $50 \%$ de fruits ou de graines) et un groupe de faunivores (alimentation essentiellement composée de proie animales - ce qui, pour les primates, correspond surtout à des insectes ou autres invertébrés). Pour chacun de ces groupes, les droites de régression ont des pentes qui les différencient significativement $(p<0,05)$. La comparaison des surfaces d'absorption du tractus digestif d'espèces de format différent implique de tenir compte de lois d'allométrie différentes, selon les types de régime: la surface absorbante varie en fonction du format corporel, proportionellement à $\mathrm{L}^{2,64}, \mathrm{~L}^{2,37}$ et $\mathrm{L}^{1,98}$, respectivement pour les folivores, les frugivores et les animalivores $(\mathrm{L}=$ longueur tête+corps). Nous avons ajouté sur la figure 3 des données (non publiées sous cette forme) concernant Homo sapiens (avec $\mathrm{L}=$ taille assis). Du seul point de vue de la morphologie du tractus digestif, ces données s'alignent sur la droite de régression obtenue pour les primates et autres mammifères à régime frugivore, incluant de fait, une grande variété de régimes, tels ceux des Sajous, des Macaques, du Chimpanzé, etc. généralement qualifiés d'omnivores (Harding et Teleki, 1981), auxquels s'ajoutent en général, à côté des fruits ou des graines qui excèdent les $50 \%$ du total ingéré, une moindre proportion de matière animale.

Les différents régimes alimentaires correspondent donc à des adaptations du tractus digestif, avec des homologies relativement précises entre espèces de même format. Les formes les plus spécialisées, consommatrices de feuillages (par exemple les deux espèces du genre Presbytis dont nous avons présenté les caractéristiques du régime) ont des appareils digestifs beaucoup plus volumineux, surtout chez les espèces de grand format impliquant une dépense énergétique pour le métabolisme propre de cet organe. Cette dépense est compensé par le faible coût énergétique de la collecte d'une nourriture abondante et peu dispersée. 


\section{Rôle de la contrainte énergétique dans l'hominisation}

Dans une série d'articles parus récemment, certains auteurs (Aiello et Wheeler, 1995 ; Leonard et Robertson, 1996, Aiello et Wheeler, 1996) ont relancé l'idée d'un éventuel rôle-clé dans l'hominisation des changements nutritionnels engendrés par les modifications climatiques à la fin du Miocène.

Selon Aiello et Wheeler, certaines adaptations biologiques de l'hominisation auraient été modelées par la nécessité d'acquérir, de préparer et de consommer une nourriture de bonne qualité, aisément digestible. Ces auteurs partent pour cela de l'observation de trois faits qui, reliés entre eux, posent problème : (1) Les hommes modernes ont un coefficient d'encéphalisation de 4 à 5 fois supérieur à celui du mammifère placentaire moyen, si l'on tient compte du rapport de la masse observée du cerveau à la masse prédite d'après Martin (1981). (2) Le cerveau est un organe dont le fonctionnement est très coûteux en énergie (son coût métabolique par kg correspond à 9 fois celui des autres parties de l'organisme). (3) Chez la plupart des mammifères, il existe une relation unique entre la masse corporelle $\left(\mathrm{P}\right.$, en $\mathrm{kg}$ ) et le métabolisme de base (MB, en kcal par jour) de la forme : $\mathrm{MB}=\mathrm{a} \cdot \mathrm{P}^{0,75}$. $\mathrm{D}^{\prime}$ où la question : quelle est la provenance du surplus d'énergie nécessité par l'encéphalisation?

L'hypothèse proposée par Aiello et Wheeler est que l'expansion du cerveau est nécessairement associée à une réduction compensatoire de la taille du tractus digestif (également grand consommateur d'énergie), donc à un régime de haute qualité aisément digestible et dense en calories (riche en graisses en protéines, pauvre en fibre). Ainsi, l'émergence du genre Homo aurait été liée à l'incorporation dans le régime d'une plus grande quantité d'aliments de haute qualité, et, quels qu'en soient les facteurs responsables, l'encéphalisation des hominidés, aurait été facilitée parce qu'un surplus d'énergie n'était plus nécessaire.

Cependant, pour justifier leur hypothèse, Aiello et Wheeler (1995) ont utilisé les données sur le tractus digestif des Primates publiées par l'un d'entre nous (Chivers et Hladik, 1980) dont nous avons présenté plus haut quelques aspects relatifs à l'allométrie. On peut d'abord s'étonner que, pour cette comparaison du genre Homo avec les autres Primates, les auteurs de l'hypothèse ne fassent référence qu'à cette première publication où seules figurent les données sur les primates non humains, en ignorant les données sur Homo sapiens publiées par la suite (Mac Larnon et al., 1986; Hladik et Chivers, 1994) et que nous avons reportées pour partie sur la figure 3 .

En fait, les différences qui apparaissent lorsqu'on compare la masse intestinale observée chez Homo sapiens à celle d'un primate de même format - prédite en fonction de l'échantillon d'espèces dont nous avons mesuré les tractus digestif —, traduisent essentiellement les différence des régimes alimentaires entre l'homme et cet échantillon de primates non-humains. Car cet ensemble de primates inclue de nombreuses formes folivores regroupées avec les primates à régime frugivore. La plus grande masse intestinale (ou son équivalent en surfaces des muqueuses mesurées) correspond à la tendance folivore de la moyenne des espèces considérées. Le raisonnement de Aiello et Wheeler est donc en grande partie circulaire, puisque basé sur cette non concordance de la taille du système

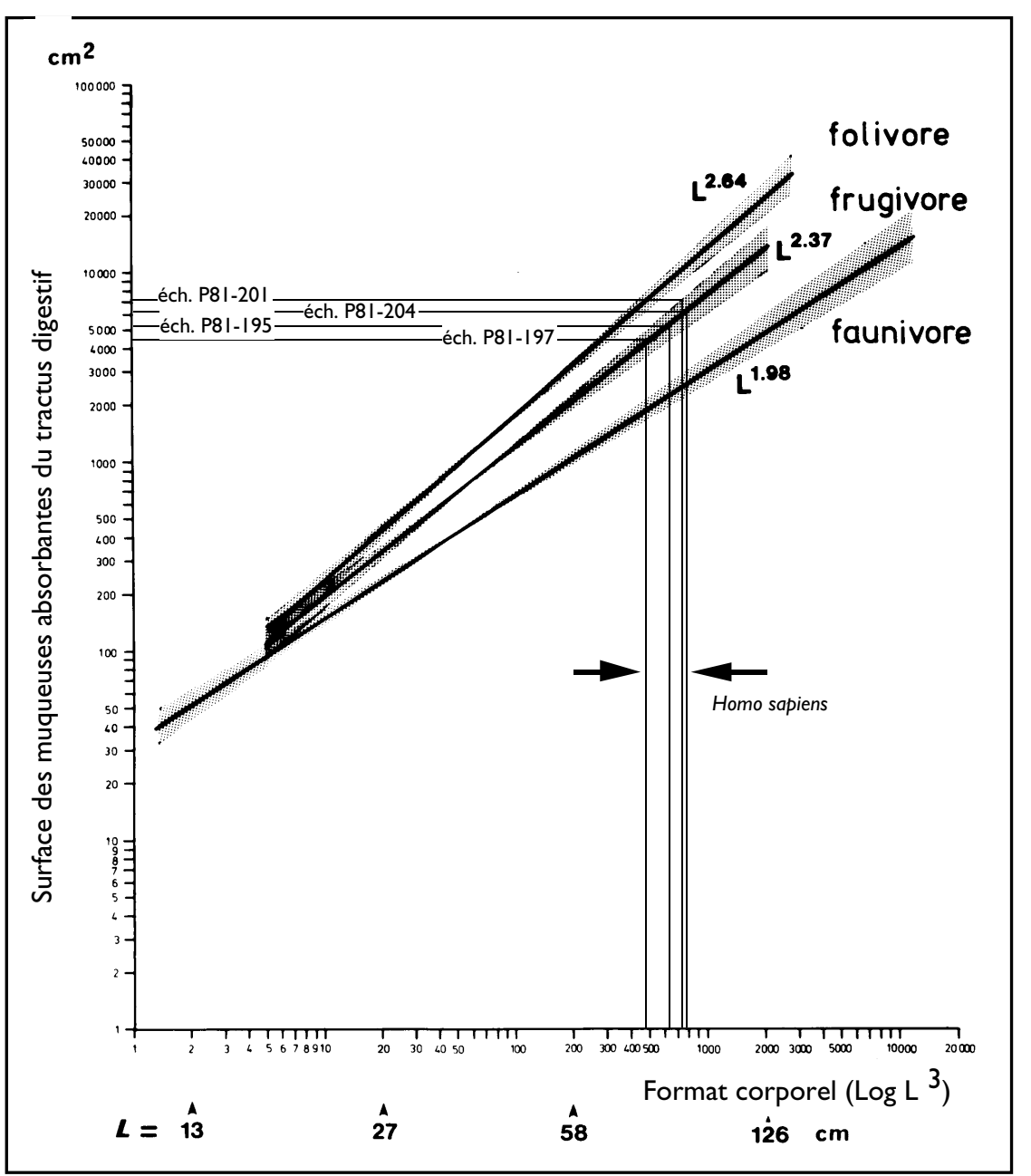

Figure 3. - Relations d'allométrie entre la surface des muqueuses absorbantes du tractus digestif et le format corporel pour trois ensembles d'espèces regroupées selon la dominante du régime alimentaire (sur un échantillon incluant 117 Primates de 50 espèces, parmi 180 mammifères; d'après Chivers et Hladik, 1980). Nous avons ajouté sur ce graphique les données concernant quatre des sujets humains étudiés par la suite, selon les mêmes méthodes, à l'Université de Cambridge (échantillons P81 de Hladik et Chivers; données non publiées sous cette forme). Les formats corporels sont exprimés, sur une échelle logarithmique, en fontion de la longueur tête+corps (L) des animaux dont on a mesuré le tractus digestif. Pour les sujets humains étudiés post-mortem, la taille assis sert de longueur de référence (L) pour une expression du format corporel $\left(10^{-3} \mathrm{~L}^{3}\right)$ comparable à celui des autres primates. 
digestif de l'Homme avec ce qu'elle devrait être, compte tenu de la morphologie des autres primates : ce qui limite la validité de leur hypothèse centrale (Hladik et al., 1999).

L'obtention d'une nourriture énergétique reste évidemment une nécessité; mais cette caractéristique énergétique n'est pas propre à la lignée des hominidés qui, comme on le voit sur la figure 3, reste proche de la médiane de la plupart des autres primates à régime frugivore ou granivore prédominant.

Les adaptations fonctionnelles du tractus digestif sont diversifiées — chez les primates comme chez les autres mammifères - , cependant un réel allègement du coût énergétique de la fonction digestive impliquerait de passer au régime carnivore, avec une surface d'absorption qui varierait comme le carré de la taille, $\mathrm{L}\left(\mathrm{L}^{1,98}\right.$, alors qu'il est respectivement de $\mathrm{L}^{2,37}$ et $\mathrm{L}^{2,64}$ pour les ensembles des frugivores et des folivores).

Le problème de l'évolution des hominidés vers le régime carnivore a déjà fait l'objet de débats (Ardrey, 1976; Ducros et Ducros, 1992). La grande diversité des régimes des populations humaines correspond à la persistance d'une morphologie digestive de type frugivore au sens large (ou omnivore) qui a permis de conserver une grande flexibilité d'adaptation. Mais l'étude comparative du tractus digestif, compte tenu des facteurs d'allométrie, ne vient en aucun cas étayer les hypothèses les plus radicales (transformation simultanée du cerveau et de l'intestin; adaptation à la carnivorie) dans la discussion sur les facteurs déterminants de l'hominisation.

\section{VALEUR ADAPTATIVE DE LA PERCEPTION GUSTATIVE}

Nous devons considérer les organes de perception biochimique, en particulier tous les éléments de la chaîne de perception gustative, comme une partie intégrante du système digestif, au même titre que la denture et le tractus intestinal. La diversification évolutive des organes de la gustation joue un rôle clé, chez les primates, dans les adaptations au régime alimentaire (Simmen, 1994). En effet, avant que le processus masticatoire ait modifié la structure physique d'un aliment, les perceptions les plus immédiates qui déterminent la prise d'un aliment ou son rejet, peuvent aussi contribuer, par la salivation, à l'efficacité de la digestion.

Si toutes les catégories biochimiques des composants alimentaires ne correspondent pas nécessairement à des stimuli clairement définis (c'est le cas, par exemple, des amidons et des graisses), il existe en revanche, parmi les composés les plus courants des végétaux et des animaux consommables, certaines classes de composés associés à des signaux dont la signification évolutive est très claire (Hladik et Simmen, 1996). Il s'agit, d'une part, des substances énergétiques à effet rapide comme beaucoup de sucres solubles, et, d'autre part, des catégories de substances souvent toxiques, comme des alcaloïdes et les thiocyanates, ou dont l'effet négatif sur l'équilibre ionique (acides forts, certains sels) ou sur l'absorption intestinale (tanins) pourrait avoir des conséquences tout aussi graves.

\section{Produits énergétiques}

Les observations sur les réponses des primates nouveau-nés (homme compris) aux stimuli alimentaires ont amené l'idée d'un fondement biologique du comportement alimentaire, et en particulier de l'existence de déterminants innés et universaux des préférences alimentaires qui passeraient par le canal de la perception gustative (Steiner 1977 ; Beauchamp et al., 1982, Steiner et Glaser, 1984).

En ce qui concerne les sucres solubles les plus fréquents dans le milieu naturel (fructose, glucose et saccharose), tous les tests comportementaux effectués sur les primates on montré une réaction positive d'acceptation des solutions, dès que la concentration atteint une valeur seuil. Ce seuil de préférence - dont on a pu montrer qu'il ne différait que très peu du seuil de réponse physiologique enregistré sur le nerf de la gustation (Hellekant et al.,1993) - a permis des comparaisons interspécifiques.

L'une des caractéristiques les plus remarquables, récemment mise en évidence chez les primates, de cette sensibilité aux solutions sucrées, est son rapport au format corporel (Simmen et Hladik, 1998). On remarque (Figure 4) qu'en dépit d'une assez large dispersion des données (imputée, pour partie, à la spécialisation extrême de certaines stratégies alimentaires), la corrélation est significative avec une droite de régression Log-Log $(\mathrm{r}=$ $0,58 ; \mathrm{p}<0,003)$ entre le poids des espèces de primates non humains et le seuil de perception du saccharose. Les espèces les plus sensibles au goût du sucre (seuils les plus bas) sont les formes les plus grandes. Homo sapiens, ne fait pas exception à la règle car il se situe dans la marge de variation à $95 \%$, sur le prolongement de la droite de régression calculée pour des primates non humains.

Cette relation d'allométrie, qui existe également pour la sensibilité des primates aux solutions de fructose, permet d'émettre une hypothèse en rapport avec les stratégies énergétiques. L'utilisation des ressources les plus denses en énergie, ainsi que nous l'avons rappelé plus haut, nécessite généralement de grands déplacements. Les espèces de peti format qui utilisent les seuls fruits à forte teneurs en sucres ont, relativement à leur poids, un bilan d'énergie qui s'équilibre à un niveau élevé. Au contraire, les espèces de grand format qui équilibrent leur bilan d'énergie à un plus bas niveau, utilisent un grand nombre de fruits d'espèces végétales très diversifiées et plus abondantes, ce qui nécessite de moindres déplacements (Hladik, 1981). L'abaissement de leur seuil de perception des sucres autorise un élargissement de l'éventail des ressources utilisables, en incluant des fruits à faible teneur en sucre (Simmen et Hladik, 1998) et le total ingéré permet la couverture de besoins énergétiques nécessairement plus élevés que ceux des primates de petit format.

Cette hypothèse permet d'interpréter la tendance globale et de situer les différentes espèces par rapport à un axe médian, indépendamment de leur format corporel. Elle permet aussi de caractériser les divergences dues aux spécialisations des régimes. En effet, les espèces qui se situent à l'écart de la droite de régression (Figure 4) sont adaptées, soit à des régimes très particuliers (par exemple, chez le Loris lent, Nycticebus coucang, l'utilisation des proies à fort goût répulsif qu'un seuil de perception élevé peut favoriser), 


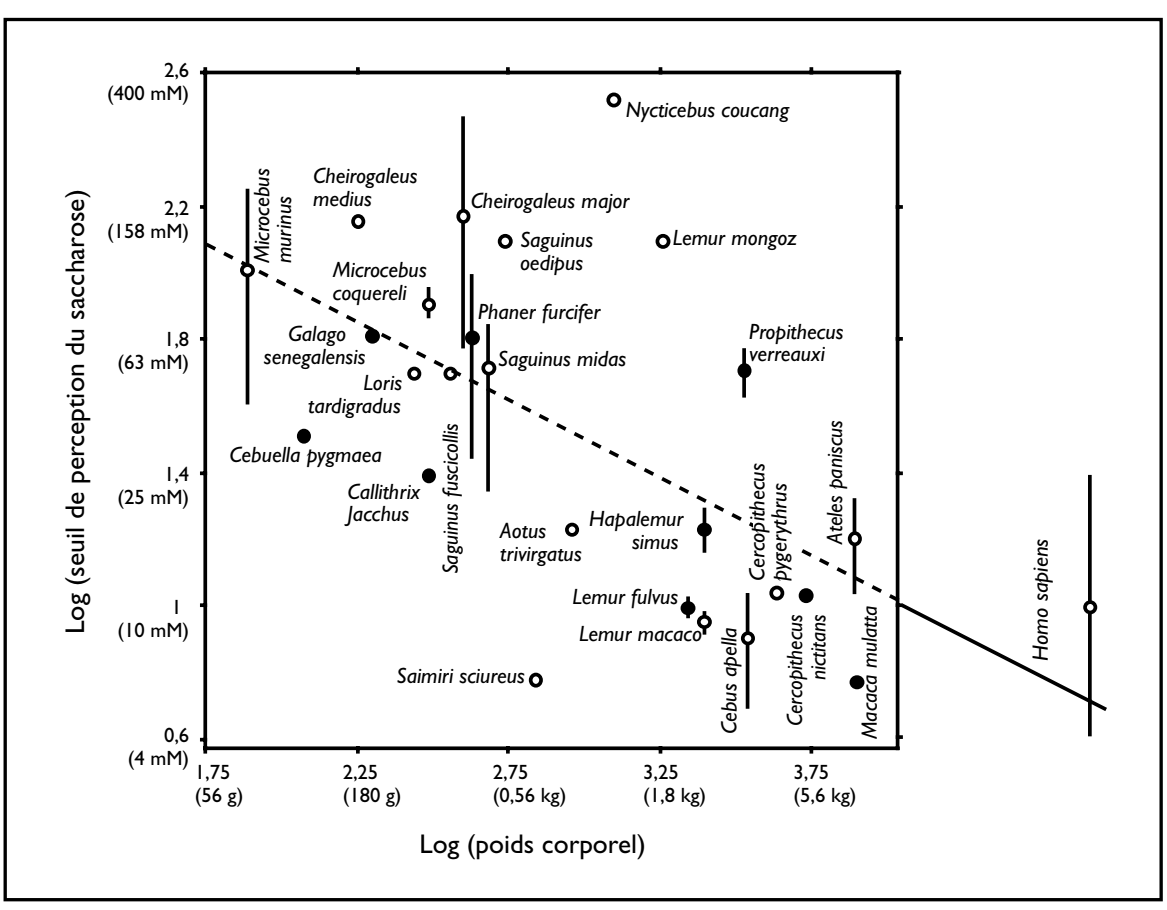

Figure 4. - Relation d'allométrie entre les seuils de perception gustative du saccharose et le poids corporel des primates non humains (d'après Simmen et Hladik, 1998) et de l'Homme. Les données concernant les formes folivores (plus de $50 \%$ de feuillages dans l'alimentation) sont positionnées par des cercles pleins ; les cercles ouverts indiquent celles des formes à dominance frugivores ou insectivores; les barres verticales correspondent à la variabilité intra-spécifique, lorsqu'elle est connue. La droite de régression (en pointillés) montre une tendance à une plus grande acuité gustative (seuils les plus bas) en relation avec les poids corporels les plus élevés. Les données concernant Homo sapiens (d'après Hladik et al., 1986) sont localisées dans la marge de probabilité $(\mathrm{p}<0,05)$ de la droite de régression calculée pour les primates non humains.

soit par des stratégies énergétiques extrêmes (par exemple chez le Singe-écureuil, Saimiri sciureus, le seuil de perception très bas favoriserait l'utilisation d'une très large game de ressources dispersées).

La variabilité du seuil de reconnaissance des sucres solubles est relativement importante chez l'humain. Des hypothèses ont été proposées pour expliquer la persistance des différences observées entre certaines populations qui vivent dans des environnement contrastés (Hladik et al., 1986; Simmen et Hladik, 1993). Par exemple, la faible acuité gustative de populations originaires des forêts tropicales pourrait résulter de la faible pression sélective d'un environnement où les fruits présentent de fortes concentrations des sucres, alors que dans les mileux plus ouverts de type sahélien, la faible teneur en sucre de la majorité des fruits peut favoriser la tendance vers un élargissement des produits consommables, par une meilleure discrimination. En fait, dans la plupart des études concernant l'espèce humaine, il apparaît une telle influence des facteurs socioculturels dans les choix alimentaires qu'il est difficile de séparer, dans la discrimination des goûts, les effets de l'apprentissage et ceux hérités de notre fond biologique de Primate.

De plus, le comportement alimentaire des Primates apparaît davantage sous la dépendance des réactions supraliminaires que du seuil de perception des solutions sucrées (Simmen, 1997) bien que peu de recherches aient été réalisées dans ce domaine. Les réponses différentielle des espèces aux solutions sucrées de concentration élevée (telles qu'on les trouve dans les aliments consommés) permettent d'interpréter les motivations dans les choix alimentaires, étant bien entendu qu'en plus d'un profil de montée en intensité plus ou moins rapide selon les espèces, les réactions supraliminaires se définissent nécessairement à partir du seuil, en fonction duquel elles varient également d'une espèce à l'autre et, éventuellement, d'une population à l'autre.

Enfin, parmi les produits alimentaires apportant de l'énergie, les plus abondants comme l'amidon et les graisses ne correspondent pas à les signaux clairement identifiés dans le registre de la gustation (Hladik et Simmen, 1996); et le goût des aliments apportant de l'énergie est souvent identifié indirectement grâce à la présence d'autres composants repérables par le sens de la gustation.

\section{Produits potentiellement toxiques}

De la même façon, les aliments toxiques sont rapidement repérés grâce à des associations (conditionnement aversif) avec des goûts qui ne sont pas nécessairement ceux des substances actives. Le conditionnement aversif s'établit à la suite d'une seule expérience (Rozin, 1990) et la néophobie (méfiance vis-à-vis des aliments nouveaux) évite généralement la consommation d'une quantité léthale d'un aliment toxique. Cela permet un apprentissage précoce (associé au malaise qui a suivi l'ingestion) et l'évitement subséquent par le dégoût acquis vis à vis de cet aliment.

Cependant, parallèlement au cas des aliments énergétiques dont la perception et la préférence de certains composants (comme les sucres) ne nécessite aucun apprentissage, il existe des catégories biochimiques potentiellement toxiques dont le goût provoque d'emblée un réaction de rejet. C'est le cas des alcaloïdes, dont beaucoup sont perçus par l'Homme comme fortement amers, ainsi que des tanins, des acides forts et de nombreux autres produits irritants dont la perception du goût peut reposer à la fois sur une stimulation biochimique des bourgeons du goût de la cavité buccale et celle des terminaisons libres (sensations douloureuses de piquant) des voies efférentes trigéminales.

En fait, il existe une forme de réflexe gusto-facial (Steiner et Glaser, 1984) qui permet aux jeunes primates d'éviter l'ingestion de ces produits et en particulier de ceux dont le goût est décrit comme amer par la grande majorité des humains. La répulsion se manifeste 
toujours chez l'adulte dès le seuil de reconnaissance - variable d'une espèce à l'autre. La relation entre ces seuils de reconnaissance des substances amères (en particulier la quinine) et la fréquence des alcaloïdes toxiques dans les environnements où ces espèces ont évolué a déjà été discutée dans un article de la présente revue (Simmen et Hladik, 1993). En dépit de cette variabilité, nous avons testé s'il pouvait y avoir, comme dans le cas de la perception des sucres, une relation au format corporel: au contraire, pour la quinine, il n'existe aucune corrélation entre seuil de perception et poids moyen pour les espèces de primates testées (Simmen et Hladik, 1998). La signification adaptative d'une bonne détection des alcaloïdes apparaît lorsqu'on considère leur toxicité potentielle, car la détection et l'évitement de ces produits de défense des végétaux dans les différents environnements nécessite une adaptation du seuil de reconnaissance fonction du risque réel de les rencontrer.

Pour les substances dont la nocivité ne se manifeste que pour d'importantes quantités ingérées et qui, tel le chlorure de sodium, qui entrent dans l'alimentation à des taux de concentration modérés, il existe, selon le contexte, une ambivalence des réactions de préférence et de rejet. Les Primates non humains trouvent généralement dans leur environnement des aliments qui permettent de couvrir leurs besoins sans aucun risque de carence. Ainsi la consommation de terre ne correspond que très rarement à une recherche de sels de sodium (voir la discussion sur la géophagie in Hladik, 1998). Chez l'Homme cette ambivalence est manifeste dans la population Inuit qui présente à la fois le seuil de reconnaissance le plus bas (très grande sensibilité) et une aversion associée au goût salé excessif, dont nous avons également discuté dans ce bulletin du déterminisme à la fois biologique et culturel (Simmen et Hladik, 1993). Les modifications successives de la perception d'une solution salée au cours de la petite enfance, observées par Beauchamp et Cowart (1985) résultent de cette plasticité de la perception des substances dont les caractéristiques, bénéfiques ou nocives, dépendent du contexte environnemental.

Des réactions positives ou négatives vis à vis des acides faibles à des concentrations supraliminaires, ont également été observées chez différentes espèces de primates (Glaser et Hobi, 1985). Plus récemment, Ungar (1995) a remarqué que les choix des primates sympatriques d'une forêt de Sumatra étaient en grande partie déterminés par le $\mathrm{pH}$ des fruits disponibles : alors qu' un Macaque (Macacafascicularis) et le Gibbon lar (Hylobates lar) consomment des fruits très acides, les Langurs (Presbytis thomasi) ne consomment que des fruits à $\mathrm{pH}$ relativement élevé. Ces Langurs consommateurs de feuillages ont une morphologie stomacale de type ruminant et leur comportement alimentaire, en relation avec la perception des acides, peut leur éviter une acidification de la poche stomacale qui détruirait la faune symbiotique indispensable à une digestion efficace.

Parmi les autres produits potentiellement toxiques à forte dose, les tanins (polyphénols) sont vraisemblablement les substances les plus répandues dans les aliments potentiels des différents écosystèmes où vivent des primates non humains, ainsi que dans les milieux où le genre Homo a évolué. Leur goût astringent caractérise, par exemple les glands du chêne ou, en beaucoup plus prononcé, celui de la pulpe immature des fruits du kaki qui surprend toujours un sujet naïf goûtant pour la première fois à ce fruit. L'étude de la perception gustative de ces substances par les primates constitue une nouvelle voie de recherche permettant de préciser à la fois les limites des adaptations possibles du comportement alimentaire des primates non humains (Simmen et al., 1999) et, chez la plupart des populations humaines qui évitent la plus grande partie des aliments contenant des tanin (sauf le thé ou le vin), dans quelle mesure l'adaptation biologique de la perception gustative peut se répercuter sur — ou interférer avec — les choix qu'imposent l'environnement psychosocial.

Cependant, chez l'homme, les résultats des travaux publiés au cours des dernières décennies portent surtout sur la perception gustative des produits perçus comme amers, sur lesquels il convient de revenir afin de préciser dans quelle mesure on peut relier les bases biologiques au comportement alimentaire observé, dans la perspective de l'évolution humaine.

\section{De la perception des amers dans la perspective de l'évolution humaine}

Chez l'homme, la relation entre le génome et le comportement alimentaire n'apparaît comme clairement démontrée que pour la perception gustative de certaines substances goûtant dans l'amer: quinine et surtout le phénylthiocarbamide et ses dérivés.

En ce qui concerne la perception de la quinine, les variations d'acuité gustative entre les différents individus d'une population découle d'une héritabilité partielle de plusieurs gènes (Fischer et Griffin, 1963; Smith et Davis, 1973). Ce polymorphisme génétique pourrait avoir joué un rôle dans l'adaptation nutritionnelle des populations humaines au cours de l'évolution. Comme dans le cas des primates non humains, un seuil élevé (correspondant à une plus grande tolérance à certains produits amers) pourrait refléter une adaptation à certains milieux ou à certains régimes alimentaires ; en particulier lorsque des produits amers antipaludéens consommés peuvent avoir un effet bénéfique. Greene $e t$ al. (1993) ont formulé l'hypothèse que les différences génétiquement induites d'acuité gustative pour la quinine permettent de réguler les ingestats de telles substances et que ce trait aurait coévolué avec le gène qui code pour la déficience en Glucose-6-phosphatedéhydrogénase dont les porteurs présentant une plus grande résistance à la malaria.

Une autre découverte de la relation entre le génome et la perception gustative chez l'homme est accidentelle: en 1931, Arthur L. Fox, un chimiste américain de la firme Dupont de Nemours, qui synthétisait des produits parmi lesquels figurait le phénylthiocarbamide (ou PTC), en laissa échapper quelques cristaux fins en suspension dans l'air. Un de ses collaborateurs en perçût l'extrême amertume, alors que pour Fox le produit semblait insipide. Il s'avéra, de fait, que le personnel du laboratoire se distribuai en deux catégories: «goûteurs» et «non goûteurs» (Fox, 1932). Les premières études sur la nature des facteurs génétiques impliqués conclurent, sur la base d'observations de la ségrégation familiale mendélienne, à l'existence d'un gène majeur (Blakeslee, 1932; Harris, 1955) — l'allèle «goûteur» étant l'allèle dominant.

Cette bimodalité phénotypique s'observe chez d' autres mammifères, notamment chez le chimpanzé (Fisher et al., 1939; Eaton et Gavan, 1965). Elle n'a pas pu être mise en 

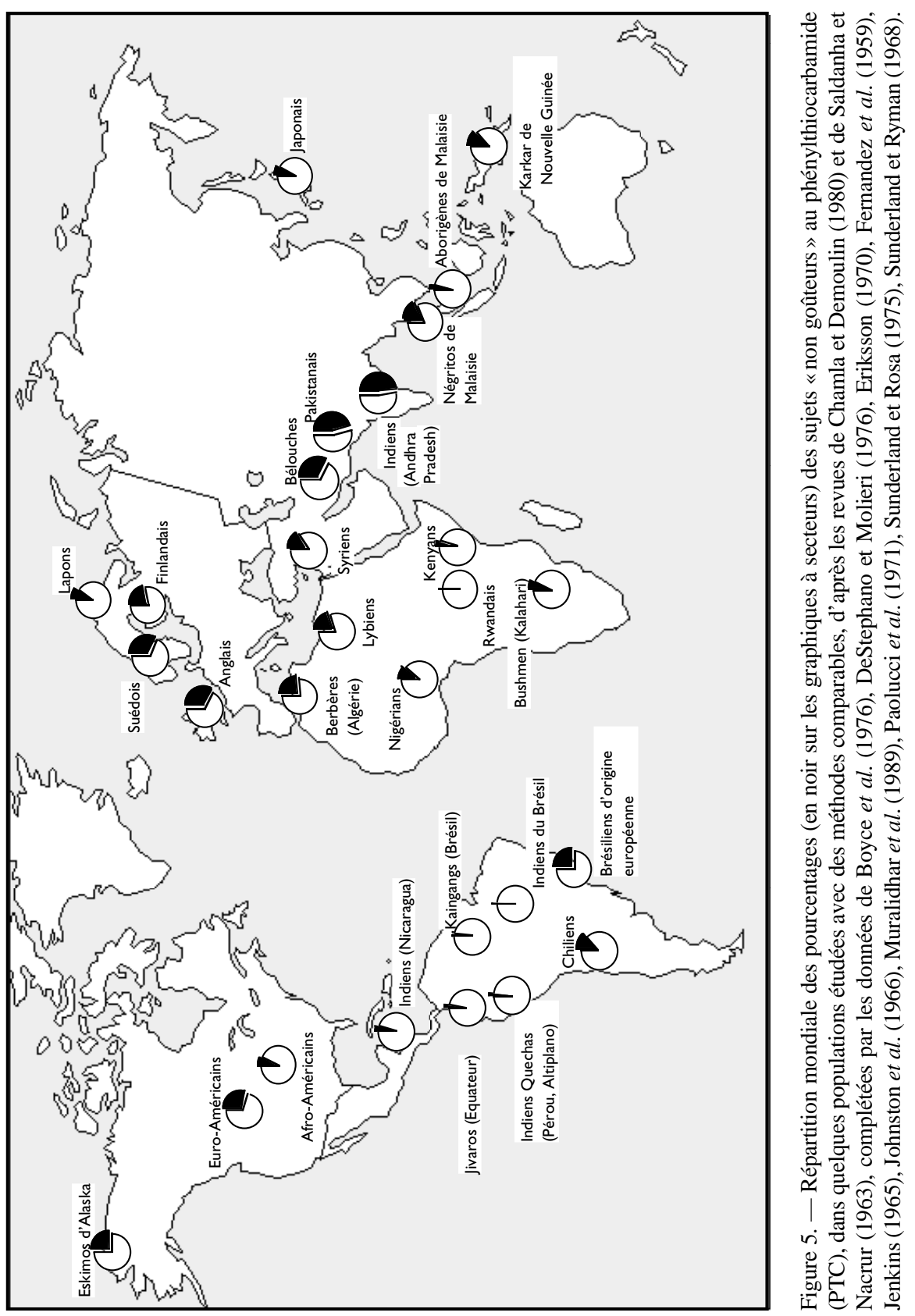

évidence chez d'autres primates; toutefois, plusieurs lignées de souris présentent des seuils de sensibilité au PTC significativement différents, ce qui pourrait correspondre au polymorphisme génétique des anthropoïdes et de l'Homme (Klein et DeFries, 1970). Du point de vue phylogénétique et évolutif, on peut s'interroger sur la signification de la persistance de ce polymorphisme bien antérieur à l'émergence des Hominidés.

En fait, chez l'Homme, on observe une relative dispersion des seuils de perception autour des modes «goûteur » et «non gô̂teur » lorsqu' on utilise la technique des dilutions successives (Blakeslee et Salmon, 1935; Harris et Kalmus, 1949), plutôt que de faire goûter directement les cristaux de PTC. Grâce aux méthodes récentes de génétique quantitative, Reddy et Rao (1989) ont étudié la sensibilité gustative au PTC chez les membres de 100 familles nucléaires indiennes; ils ont constaté que la distribution du caractère était liée à $96 \%$ à un gène majeur (avec dominance incomplète) ainsi qu'à la somme de l'influence d'autres gènes (qui explique la distribution autour de chaque mode). Morton et al. (1981), utilisant un autre modèle (enfants de jumeaux monozygotes), accordent, dans leur conclusion, une plus grande importance aux facteurs environnementaux (entre $30 \%$ et $45 \%$ ) avec un important effet de transmission maternelle. Enfin, Olson et al. (1989), se basant sur l'étude de 120 familles américaines, ont proposé un modèle à deux gènes sur 2 locus : un locus où un gène coderait pour la perception du $\mathrm{PTC}$, et un autre où un gène coderait pour l'acuité gustative en général. Ce modèle semble conforme aux observations récentes sur la relation entre la sensibilité au PTC et la sensibilité gustative à d'autres produits (Franck et Korchmar, 1985; Bartoshuk, 1993).

Se basant sur l'importante variabilité des seuils de perception et sur l'apparente variété de perception de l'amer au dessus de ce seuil, des chercheurs de l'Université de Yale ont, en outre, émis l'hypothèse de l'existence de deux catégories au sein du groupe de goûteurs: les goûteurs et les super-goûteurs (Bartoshuk, 1993). Ainsi aux USA, il y aurait $25 \%$ de «non goûteurs», $25 \%$ de «super-goûteurs » et $50 \%$ de «goûteurs ». Les goûteurs seraient les hétérozygotes Tt, et les supergoûteurs les homozygotes TT du phénotype goûteur. Anatomiquement, les supergoûteurs possèdent une nombre beaucoup plus important de bourgeons du goût au niveau des papilles fongiformes, elles-même présentant chez ces sujets des caractéristiques anatomiques particulières (Miller et Reddy, 1990; Reddy et al., 1993).

Des études de liaison génétique ont montré, par ailleurs, que le gène codant pour la sensibilité au PTC est transmis en concomitance avec celui qui code pour le groupe sanguin Kell. Ce dernier étant localisé sur le chromosome 7q, cela signifie que le locus PTC se trouve également sur ce chromosome (Coneally et al., 1976).

La sensibilité différentielle au PTC a été amplement utilisée comme marqueur génétique des populations humaines et a donné lieu à une multitude de travaux. La proportion de «goûteurs» et de «non goûteurs» varie d'une population à l'autre (Figure 5). La fréquence du phénotype «non goûteur» est inférieure à $3 \%$ chez les amérindiens (Paolucci et al. 1971 ; Sunderland et Ryman, 1968 ; Fernandez et al., 1957). On observe des proportions de l'ordre de $5 \%$ à $10 \%$ dans de nombreuses populations d'Afrique subsaharienne, chez les afro-américains et certaines populations asiatiques. Des proportions plus importantes 
(entre $20 \%$ et $30 \%$ ) se trouvent en Europe (ainsi que chez les descendants d'européens en Amérique), en Afrique du Nord et au Moyen Orient. Les fréquences les plus élévées s'observent sur le continent indien (47\%, par exemple, dans l'Andhra Pradesh ; Muralidhar et al., 1989).

Cette répartition particulière a été expliquée (Greene, 1974) par le fait que les goûteurs au PTC sont également goûteurs pour toute une famille de composés: la thiourée, le PROP (6-n-propylthio-uracile) — qu' on utilise désormais pour les tests, de préférence au PTC — et plus d'une quarantaine d'autres produits, incluant des substances naturelles qui ont des effets antithyroïdiens ou très toxiques pour certaines d'entre elles. En conséquence, la répartition observée pourrait être le reflet d'une sélection des gènes «goûteurs » dans un milieu ou il y a une forte concentration de produits incluant le radical $\mathrm{N}-\mathrm{C}=\mathrm{S}$ (Boyd, 1950), en particulier en Amérique du Sud et en Afrique subsaharienne, dans des régions à faible concentration d'iode où les populations sont particulièrement sujettes au goitre.

Dans les populations pour lesquelles le manioc est un aliment de base, les «goûteurs », en évitant les formes les plus amères, pourraient limiter les effets antithyroïdiens des glycosides cyanogéniques. L'équilibre qui s'établit avec les génotypes présentant une résistance à la malaria - lorsque les effets néfastes de l'anémie falciforme sont atténués par de faibles doses de cyanogènes (Jackson, 1996) — n'empêche pas, dans certaines régions, que le phénotype «non-goûteur» ait quasiment disparu.

La relation entre la sensibilité aux produits amers et les préférences/aversions alimentaires a été explorée, notamment par Fischer et al. (1961) et Anliker et al. (1991): plus la sensibilité est grande, plus grand est le nombre de produits perçus comme peu palatables parmi une liste d'aliments familiers, ce qui va dans le sens des spéculations évolutionistes exposées ci-dessus. Ce phénomène n'a été mis au jour que pour la sensibilité gustative aux produits amers. Aucune relation n'a pu être établie entre la sensibilité aux produits sucrés ou aux substances goûtant dans le salé et les rejets ou les préférences alimentaires (Fisher et Griffin, 1965).

C'est sur cette base qu'ont été entrepris une série de travaux récents pour déterminer la relation entre la sensibilité gustative aux produits amers (en particulier le PROP et ses dérivés) et la consommation d'aliments goitrogènes, notamment les crucifères dont l'intérêt renouvelé réside dans leur composition en agents protecteurs vis à vis de certaines pathologies incluant des cancers. Malheureusement, les études entreprises (Niewind $e t$ al., 1988 ; Mattes et Labov, 1989 ; Jerzsa-Latta et al., 1990) n'ont pas permis de conclure, sur le rôle éventuel les marqueurs génétiques du goût dans la consommation de légumes crucifères, ceci peut-être pour des raisons méthodologiques qui ne tiendraient pas suffisamment compte des facteurs socioculturels dans le déterminime des choix alimentaires. Les goûteurs au PROP trouvent plus amers la caféine (Hall et al., 1975), la saccharine (Bartoshuk, 1979) et le chlorure de potassium (Bartoshuk et al., 1988). Ils trouvent également plus amer l'alcool éthylique en solution à 10\% (Bartoshuk et al., 1993). Le goût sucré de certains édulcorants tels que la saccharine, ou la neohesperidine dihydrochalcone paraît renforcé chez les goûteurs (Gent et Bartoshuk, 1983).
Mais ce sont les relations entre sensibilité aux amers et la valeur hédonique qui peuvent expliquer certains choix préférentiels. Looy et Weingarten (1992) ont montré, tant chez l'adulte que chez l'enfant, que la sensibilité au PROP pouvait conditionner la préférences pour le saccharose en altérant la qualité perçue pour ce sucre à forte concentration. Ains les non goûteurs au PROP accordent une forte valeur hédonique (niveau de plaisir gustatif) pour ce sucre, alors que les goûteurs qui le perçoivent comme plus sucré, éprouvent un certain déplaisir. Cela rejoint le paradoxe apparent de la faible acuité gustative des Pygmées vis-à-vis sucres, opposée à leur forte motivation pour la recherche du miel sauvage, et pour le prestige attribué à cette substance dans leur système social (Hladik et Bahuchet, 1994).

Il apparaît donc que les goûteurs et les non-goûteurs, et, vraisemblablement, d'autres catégories de personnes que d'autres facteurs différencieraient dans leurs modes de perception, vivent dans des univers gustatifs différents.

\section{CONCLUSIONS}

L'évolution des primates dans différents environnements implique une adaptation des régimes alimentaires reposant à la fois sur la morphologie de l'appareil digestif et sur l'équipement sensoriel. Ainsi, les seuils de perception gustative pour des substances du milieu naturel hautement énergétiques ou pour des produits toxiques, reflètent à la fois la composition des différents milieux (leur richesse en tanins, alcaloïdes, etc.) et les stratégies adaptatives portant, en particulier, sur le niveau d'équilibre du bilan énergétique. Le format corporel apparaît comme jouant un rôle déterminant dans les équilibres possibles en fonction des régimes; et les relations d'allométrie avec la perception gustative résulteraient des pressions de sélection qui s'exercent sur l'ensemble des espèces.

Nous avons présenté quelques exemples de milieux où la co-exitence en sympatrie de plusieurs espèces de Primates permet d'analyser ces processus à partir d'un modèle du comportement alimentaire dans lequel nous pouvons inclure les espèces des genres Autralopithecus et Homo, sans ignorer que l'émergence de la culture en a partiellement gommé les contours en lui ajoutant de nouvelles dimensions.

Pour les populations humaines actuelles, si l'on n'a pu montrer avec certitude, à ce jour, que des liens entre la possibilité de discriminer certains produits amers et les gènes qui codent pour la sensibilité gustative correspondante, l'exploration de nos caractéristiques biologiques reste en grande partie à réaliser, en particulier dans le domaine de la perception des tannins, produits les plus abondants dans les aliments potentiels des divers milieux naturels auquels toutes les formes qui nous ont précédées ont été confrontées. 


\section{BIBLIOGRAPHIE}

Aiello (L.C.), Wheeler (P.), 1995. The expensive-tissue hypothesis. The brain and the digestive system in human and primate evolution. Current Anthropology, 36: 199-221.

Aiello (L.C.), Wheeler (P.), 1996. Reply to Leonard and Robertson. Current Anthropology, 37 : 128-129.

Amerasighe (F.P.), VANCUYlenberg (B.W.B.), HLAdik (C.M.), 1971. Comparative histology of the alimentary tract of Ceylon primates in correlation with the diet. The Ceylon Journal of Science, Biological Sciences, 9: 75-87.

Anliker (J.A.), Bartoshuk (L.), FerRis (A.M.), Hooks (L.D.) 1991. Children's food preferences and genetic sensitivity to the bitter taste of 6- $n$-propylthiouracil (PROP). American Journal of Clinical Nutrition, 54 : 316-320.

ARDREY (R.), 1976. The hunting hypothesis. A personal conclusion concerning the evolutionary nature of man. Atheneum, New-York.

BARTOSHUK (L.M.), 1979. Bitter tasre of saccharin related to the genetic ability to taste the bitter substance 6-n-propylthiouracil. Science. 205 : 934-935.

BARTOSHUK (L.M.), 1993. Genetic and pathological taste variation: what can we learn from animal models and human disease. Ciba Foundation Symposia, 179: 251-262.

Bartoshuk (L.M), Conner (E.), Grubin (D.), 1993. PROP supertasters and the perception of ethyl alcohol. Chemical Senses. 18: 526-527.

Bartoshuk (L.M), Rifkin (B.), Marks (L.E.), Hooper (J.E.), 1988. Bitterness of $\mathrm{KCl}$ and benzoate : related to genetic status for sensitivity to PTC/PROP. Chemical Senses. 13: 517-528.

Beauchamp (G.K.) Cowart (B.J.), 1985. Congenital and experiential factors in the development of human flavor preferences, Appetite, $6: 357-372$

BeAuChamp (G.K.), Bertino (M.), Moran (M.), 1982.. Sodium régulation : sensory aspects. Journal of the American Dietetic Association, 80 : 40-46.

BlakesleE (A.F.), SaLmon (T.N.), 1935. Genetics of sensory thesholds : individual taste reactions for different substances. Proceedings of the National Academy of Sciences, USA, 21 : 84-90

BlakesLeE (A.F.), Fox (A.L.), 1932. Our different taste world. Journal of Heredity, 23 : 97-110.

BLAKESLEE (A.F.), 1932. Genetics of sensory thresholds : Taste for phenylthiocarbamide. Proceedings of the National Academy of Sciences, USA, 18: 120-130.
Boyce (A.J.), Harrison (G.A.), Platt (C.M.), Hornabrook (R.W.), 1976. Association between PTC taster status and goitre in a Papua New Guinea population. Human Biology, 48: 769-

BoyD (W.C.), 1950. Taste reactions to anti-thyroid substances. Nature, 112: 153 .

Chamla (M-C.), Demoulin (F.), 1980. La sensibilité gustative à la thiourée chez les berbères chaouîas de l'Aurès (Bouzina, Algérie). L'Anthropologie, 84 : 619-632.

Chivers (D.J.), HladiK (C.M.), 1980. Morphology of the gastrointestinal tract in primates : Comparisons with other mammals in relation to diet. Journal of Morphology, 166: 337-386.

Coneally (P.M.), Dumont-Driscoll (M.), HuntZinger (R.S.), NAnCe (W.E.), Jackson (C.E.), 1976 Linkage relations of the loci for kell and phenylthiobarbamide (PTC) taste sensitivity. Human Heredity, 26: 267-271.

Cuvier (G.), 1805. Leçons d'anatomie comparée. Crochard, Paris.

De Stefano (G-F.), Molieri (J.J.), 1976. P.T.C. tasting among three indian groups of Nicaragua. American Journal of Physical Anthropology, 44 : 371-374.

Ducros (J.), Ducros (A.), 1992. Le singe carnivore: la chasse chez les primates non humains Bulletins et Mémoires de la Société d'Anthropologie de Paris, nouvelle série, 4: 243-264.

Eaton (J.W.), Gavan (J.A.), 1965. Sensitivity to P-T-C among Primates. American Journal of Physical Anthropology, 23: 381-388

Eriksson (A.W.), Fellman (J.), Forsius (H.), Lehmann (W.), 1970. Phenylthiocarbamide tasting ability among Lapps and Finns. Human Heredity, 20: 623-630.

Fernandez (J.L.), Junqueira (P.C.), Kalmus (H.), Ottensooser (F.), Pasqualin (R.), Wishart (P.), 1957. P.T.C. thresholds, colour vision, and blood factors of Brazilian Indians. Annals of Human Genetics, 22: 16-21.

Fischer (R.), Griffin (F.), England (S.), Garn (S.M.), 1961. Taste thresholds and food dislikes. Nature, 191: 1328 .

FISCHER (R.), GRIFFIN (F.), 1963. Quinine dimorphism: a cardinal determinant of taste sensitivity. Nature, 200 : 343-347.

Fisher (R.A.), Ford (E.B.), HuXley (J.), 1939. Taste-testing the Antropoid Apes. Nature, 144 : 750

Fox (A.L.), 1932. The relationship between chemical constitution and taste. Proceedings of the National Academy of Sciences (Washington), 18: 115-120. 
Frank (R.A.), Korchmar (D.L.), 1985. Gustatory processing differences in PTC tasters and nontasters : a reaction time analysis. Physiology \& Behavior, 35: 239-242.

Garine (I. de), Harrison (G.A.), (Eds) 1988. Coping with Uncertainty in Foos Supply. Clarendon Press, Oxford.

Gent (J.F.), BARToshuK (L.M.) 1983. Sweetness of sucrose, neohesperidin, dihydrochalcone, ans saccharin is related to genetic ability to taste the bitter substance 6- $n$-propylthiouracil. Chemical Senses 7: 265-272.

Glaser (D.), Hobi (G.) 1985. Taste responses in primates to citric and acetic acid. International Journal of Primatology, 6: 395-398.

GREENE (L.S.), 1974. Physical growth and development, neurological maturation and behavioral functioning in two Ecuadorean Andean communities where goiter is endemic. II PTC taste sensitivity and neurological maturation. American Journal of Physical Anthropology, 41 : $139-151$

Greene (L.S.), McMahon (L.), DiLorio (J.), 1993. Co-evolution of glucose-6-phosphate dehydrogenase deficiency and quinine taste sensitivity, Annals of Human Biology, 20 : 497500.

Hall (M.J.), Bartoshuk (L.M.), Cain (W.S.), Stevens (J.C.), 1975. PTC taste blindness and the taste of caffeine, Nature, 253: 442-443.

Harding (R.S.O), Teleki (G.), (Eds) 1981. Omnivorous Primates: Gathering and Hunting in Human Evolution. Columbia University Press, New York.

HARRIS (H), 1955. An introduction to Human Biochemical Genetics. Eugeneics Laboratory Memoirs $\mathrm{n}^{\circ}$ 37. London Galton Laboratory, Cambridge University Press.

Harris (H.), Kalmus (H.), 1949. The measurement of taste sensitivity to phenylthiourea (PTC). Annals of Eugenics, $15: 24-31$

Hellekant (G.), Hladik (C.M.), Dennys (V.), Simmen (B.) Roberts (T.W.), Glaser (D.) Dubois (G.), Walters (D.E.), 1993. On the sense of taste in two Malagasy primates (Microcebus murinus and Eulemur mongoz). Chemical Senses, 18: 307-320.

HLAdiK (A.), HLADiK (C.M.), 1969. Rapports trophiques entre végétation et Primates dans la forêt de Barro Colorado (Panama). La Terre et la Vie, 23: 25-117.

HLADIK (C.M.), 1967. Surface relative du tractus digestif de quelques Primates, morphologie des villosités intestinales et corrélations avec le régime alimentaire. Mammalia, 31 : 120-147.

HLADIK (C.M.), 1977. A comparative study of two sympatric species of leaf monkeys: Presbytis entellus and Presbytis senex. In: T.H. Clutton-Brock (Ed.), Primate Ecology: Studies of
Feeding and Ranging behaviour in Lemurs, Monkeys, and Apes, Academic Press, London, pp. 323-353.

HLADIK (C.M.), 1981. Diet and the evolution of feeding strategies among forest primates. In : R.S.O Harding et G. Teleki (Eds.), Omnivorous Primates. Gathering and Hunting in Human evolution.Columbia University Press, New York, pp. 215-254.

HLADIK (C.M.), 1998. Aliments et médicaments : Des «traditions » chez les chimpanzés et de leurs interprétations. In: A et J. Ducros, F. Joulian, (Eds.), La Culture est-elle naturelle? Histoire, Epistémologie et Applications récentes du Concept de Culture. Editions Errance, Paris, pp. $151-161$.

HLAdiK (C.M.), BAhUChEt (S.), 1994. Perception and utilization of rain forest fruits and honey by the Aka Pygmies (Central African Republic). In: B. Thierry, J.R. Anderson et J.J. Herrenschmidt (Eds.), Selected Proceedings of the XIVth Congress of the International Primatological Society. Volume I. Editions de l'Université Louis Pasteur, Strasbourg, pp. $155-159$.

HLADIK (C.M.), Chivers (D.J.), 1994. Foods and the digestive system. In : D.J. Chivers et M. Langer (Eds), The Digestive System in Mammals : Food, Form, Function and Evolution. Cambridge University Press, pp. 65-73.

Hladik (C.M.), Chivers (D.J.), Pasquet (P), 1999 (sous presse). On diet and gut size in non-human primates and humans: is there a relationship to brain size?, Current Anthropology.

HLADiK (C.M.), GuEguen (I.), 1974. Géophagie et nutrition minérale chez les primates sauvages. Comptes Rendus des Scéances de l'Académie des Sciences, Paris, série III, 279: 1393-1396.

HLADIK (C.M.). HladiK (A.), 1972. Disponibilités alimentaires et domaines vitaux des Primates à Ceylan. La Terre et la Vie, 26 : 149-215.

Hladik (C. M.), Hladik (A.), Bousset (T.), Valdebouze (P.), Viroben (G.), Delort-Laval (J.), 1971 Le régime alimentaire des Primates de l'île de Barro Colorado (Panama). Folia Primatologica, $16: 85-122$.

Hladik (CM), Robbe (B), Pagezy (H), 1986. Sensibilité gustative différentielle des population Pygmées et non Pygmées de forêt dense, de soudaniens et d'Eskimos, en rapport avec l'environnement biochimique. Comptes Rendus des Séances de l'Académie des Sciences, Paris, série III, 303 : 453-458.

Hladik (C.M.), Simmen (B.), 1996. Taste perception and feeding behavior in nonhuman primate and human populations. Evolutionary Anthropology 5 : 58-71.

JACKSON (F.L.C.), 1996. Les conséquences bioculturelles de la consommation du manioc (Maniho esculenta) sur le métabolisme et la micro-évolution de l'Homme. In : C.M. Hladik, A. Hladik, H. Pagezy, O.F. Linares, G.J.A. Koppert et A. Froment (eds), L'alimentation en forêt tropicale. 
Interactions bioculturelles et perspectives de développement. UNESCO, Paris, pp. 511536.

JENKINS (T.), 1965. Ability to taste phenylthiocarbamide among Kalahari Bushmen and Southern Bantu. Human Biology, 37: 371-374.

Jerzsa-Latta (M), Krondl (M.), Coleman (P.), 1990. Use and perceived attributes of cruciferous vegetables in terms of genetically-mediated taste sensitivity. Appetite, 15: 127-134.

Johnston (F.E.), Hertzog (K.P.), Malina (R.M.),1966. Phenylthiocarbamide taste sensitivity and its relationship to growth variation. American Journal of Physical Anthropology, 24: 253256

Kay (R.F.), Hylander (W.L.), 1978. The dental structure of mammalian folivores with special reference to primates and Phalangeroidea (Marsupiala). In: G.G. Montgomery (Ed), The ecology of arboreal folivores, Smithsonian Institution Press, Washington, D.C., pp. 173191

KLein (T.W.), DeFries (J.C.), 1970. Similar polymorphism of taste sensitivity to PTC in mice and men. Nature, 225 : 555-557.

Krondl (M.), Coleman (P.), Wade (J.), Milner (J.), 1983. Twin study examining the genetic influence on food selection. Human Nutrition: Applied Nutrition, 37: 189-198.

LeONARD (W.L.), RoberTson (M.L.), 1996. On diet, energy metabolism, and brain size in human evolution. Current Anthropology, 37: 125-128.

Looy (H.), Weingarten (H.P.), 1992. Facial expressions and genetic sensitivity to 6- $n$-Propylthiouracil predict hedonic response to sweet. Physiology and Behavior, 52:75-82

LuCAS (P.W.), 1994. Categorization of food items relevant to oral processing. In: D.J. Chivers et P.M. Langer (Eds), The Digestive System in Mammals : Food, Form, Function and Evolution, Cambridge University Press : 197-218.

Mac Larnon (A. M.), Martin (R.D.), Chivers (D.J.), Hladik (C.M.), 1986. Some aspects of gastrointestinal allometry in Primates and other Mammals. In: M. Sakka (Ed.), Définition et Origines de l'Homme. Editions du CNRS, Paris, pp. 293-302.

Martin, (R.D.), 1981. Relative brain size and basal metabolic rate in terrestrial mammals, Nature, 293: 57-60.

Mattes (R). et Labov (J.), 1989. Bitter taste responses to phenylthiocarbamide are not related to dietary goitrogen intake in human beings. Journal of the American Dietetic Association, 89 : 692-694..
Milier (I.J.), Reddy (F.E.) JR., 1990. Variations in human taste bud density and taste intensity perception. Physiology \& Behavior, 47 : 1213-1219.

Morton (C.C.), Cantor (R.M.), Corey (L.A.), Nance (W.E.), 1981. A genetic analysis of taste threshold for phenylthiocarbamide. Acta Genet Med Gamellol, 30 : 51-57.

Muralidhar (B.), Goud (J.D.), MurTy (J.S.), 1989. Genetic structure of three Naikpod subpopulation of Andhra Pradesh, India. American Journal of Physical Anthropology, 80 : 41-47.

Niewind (A.), Krondl (M.), ShrotT (M.), 1988. Genetic influences on the selection of brassica vegetables by elderly individuals. Nutrition research, $8: 13-20$.

Olson (J.M.), Boehnke (M.), Neiswanger (K.), Roche (A.F.), Siervogel (R.M.), 1989. Alternative genetic models for the inheritance of the phenylthiocarbamide (PTC) taste deficiency. Genetic Epidemiology, 6: 423-434

Paolucci (A.M.), Ferro-Luzzi (A.), Modiano (G.), Morpurgo (G.), Kanashiro (K.), 1971. Taste sensitivity to phenylthiocarbamide (PTC) and endemic goiter in the Indian natives if Peruvian highlands. American Journal of Physical Anthropology, 34 : 427-430.

RedDy (BM), Rao (DC), 1989. Phenylthiocarbamide Taste Sensitivity Revisited: Complete Sorting Test Supports Residual Family Resemblance. Genetic Epidemiology, 6: 413-421.

Reddy (F.E.), Bartoshuk (L.M.), Miller (IJ.), Duffy (V.B.), Yanagisawa (K.), 1993. Relationship among papillae, taste pores and 6-n-propylthiouracile (PROP) suprathreshold taste sensitivity. Chemical senses, 18: 618-619.

Rozin (P.), 1990. Acquisition of stable food preferences. Nutrition Reviews, 48: 106-113.

SALDANHA (P.H.), NACRUR (J.), 1963. Taste thresholds for phenylthiourea among Chileans. American Journal of Physical Anthropology, 21 : 113-119.

Simmen (B.), 1994. Taste discrimination and diet differenciation among New World primates. In D.J. Chivers, P.M. Langer (Eds), The Digestive System in Mammals : Food, Form, Function and Evolution, Cambridge University Press, pp. 150-165.

Simmen (B.), 1997. Food preferences in neotropical primates in relation to taste sensitivity. In: $\mathrm{H}$ Macbeth (Ed.), Food Preferences and taste: Continuity and Change. Berghahn books, Oxford, pp. 28-37.

Simmen (B.), HLAdiK (C.M.), 1993. Perception gustative et adaptation à l'environnement nutritionnel des Primates non humains et des population humaines. Bulletins et Mémoires de la Société d'Anthropologie de Paris, n. s., 5 : 343-354.

Simmen (B.), HLAdiK (C.M.), 1998. Sweet and bitter taste discrimination in primates : scaling effects across species. Folia Primatologica, 69: 129-138. 
Simmen (B.), Josseaume (B.), Atramentowicz (M.), 1999. Frugivory and taste responses to fructose and tannic acid in a prosimian primate and a didelphid marsupial. Journal of Chemical Ecology, 25 : 331-346.

Smith (S.E.), Davies (P.D.O.), 1973. Quinine taste thresholds: a family study and a twin study. Annals of Human Genetics, $37: 227-232$.

Steiner (J.E.), Glaser (D.), 1984. Differential behavioral responses to taste stimuli in nonhuman primates. Journal of Human Ecology, 13 : 709-723.

STEINER (J.E.), 1977. Facial expressions of the neonate infant indicating the hedonics of food-related chemical stimuli. In: J.M. Weiffenbach (Ed.), Taste and developement. Epigenetic of sweet preferences, NIH-DHEW, Bethesda, pp. 173-189.

Sunderland (E.), Ryman (R.), 1968. P.T.C. thresholds, blood factors, colour vision and fingerprints of Jivaro Indians in Eastern Ecuador. American Journal of physical Anthropology, 28 : 339344

Sunderland (E.), Rosa (P.J.), 1975. The ability to taste phenylthiocarbamide among the tripolitanians, Cyrenaicans, and Fezzanites of Libia and the Kikuyu, Kamba, and Taita of Kenya. Human Biology, 47 : 473-481.

UNGAR (P.S), 1995. Fruit preferences of four sympatric primate species at Ketambe, Northern Sumatra, Indonesia. International Journal of Primatology, 16: 221-245.

Wrangham (R.W.), 1977. Feeding behaviour of chimpanzees in Gombe National Park, Tanzania. In: T.H. Clutton-Brock (Ed.), Primate Ecology: Studies of Feeding and Ranging behaviour in Lemurs, Monkeys, and Apes. Academic Press, London, pp. 503-538.

Recu le 12/05/1998, accepté le 10/09/1999. 